\title{
TOPOLOGICAL SPACES WHOSE BAIRE MEASURE ADMITS A REGULAR BOREL EXTENSION
}

\author{
HARUTO OHTA AND KEN-ICHI TAMANO \\ Dedicated to Professor Yukihiro Kodama on his 60 th birthday
}

\begin{abstract}
A completely regular, Hausdorff space $X$ is called a Maŕík space if every Baire measure on $X$ admits an extension to a regular Borel measure. We answer the questions about Mařík spaces asked by Wheeler [29] and study their topological properties. In particular, we give examples of the following spaces: A locally compact, measure compact space which is not weakly Bairedominated; i.e., it has a sequence $F_{n} \downarrow \varnothing$ of regular closed sets such that $\bigcap_{n \in \omega} B_{n} \neq \varnothing$ whenever $B_{n}$ 's are Baire sets with $F_{n} \subset B_{n}$; a countably paracompact, non-Mařík space; a locally compact, non-Maŕík space $X$ such that the absolute $E(X)$ is a Mařik space; and a locally compact, Mařik space $X$ for which $E(X)$ is not. It is also proved that Michael's product space is not weakly Baire-dominated.
\end{abstract}

\section{INTRODUCTION}

All spaces are assumed to be completely regular, Hausdorff spaces. Unless otherwise specified, measures are finite, nonnegative, $\sigma$-additive measures. A Baire (Borel) measure on a space $X$ is a measure defined on the $\sigma$-algebra $\mathrm{Ba}(X)$ of Baire sets $(\mathrm{Bo}(X)$ of Borel sets) of $X$. A (finitely additive) measure $\mu$ defined on an algebra $\mathscr{A}$ containing all closed sets is called regular if for each $A \in \mathscr{A}, \mu(A)=\sup \{\mu(F): F \subset A, F$ is closed $\}$. In [29, $\S 9]$, Wheeler fully reviewed a number of interesting topics relating to the problem of when a Baire measure can be extended to a regular Borel measure. In particular, he defined a space $X$ to be a Mařik space if every Baire measure on $X$ admits an extension to a regular Borel measure, and asked several questions thereupon.

This paper falls into two parts. In the first part, $\S \S 2$ and 3 , we answer some of his questions. In the second part, $\S 4$, we study how Mařík spaces are preserved under various topological operations. Before stating his questions, we recall some definitions and show which spaces are Maŕik spaces. A countably paracompact space is a space each of whose countable, open covers has a locally finite, open refinement. A space $X$ is said to be (weakly) cozero-dominated

Received by the editors June 27,1988 .

1980 Mathematics Subject Classification (1985 Revision). Primary 54C50, 28C15; Secondary $54 \mathrm{G} 20$.

Key words and phrases. Maŕík space, countably paracompact, locally compact, absolute, Michael line, Baire measure, Borel measure, regular Borel extension, measure compact. 
if for each decreasing sequence $\left\{F_{n}\right\}_{n \in \omega}$ of (regular) closed sets in $X$ with $\bigcap_{n \in \omega} F_{n}=\varnothing$ (we write this situation symbolically as $F_{n} \downarrow \varnothing$ ), there exists a sequence $\left\{U_{n}\right\}_{n \in \omega}$ of cozero-sets in $X$ such that $F_{n} \subset U_{n}$ for each $n \in \omega$ and $U_{n} \downarrow \varnothing$. Here, a regular closed set is a set that is the closure of its interior. If cozero-sets are relaxed to Baire sets, then $X$ is said to be (weakly) Bairedominated. A Baire measure $\mu$ on a space is called $\tau$-additive if, whenever a net $\left\{Z_{\alpha}\right\}_{\alpha \in A}$ of zero-sets decreases to a zero-set $Z, \mu(Z)=\inf \left\{\mu\left(Z_{\alpha}\right): \alpha \in A\right\}$. A space $X$ is called measure compact, abbreviated as $\mathrm{MC}$, if every Baire measure on $X$ is $\tau$-additive (cf. $[29, \S 8]$ ). The relationship of these spaces to a Mařik space is summarized as the following:
(1) normal and
(2) countably compact

countably paracompact

(3) cozero-dominated

(6) weakly

(4) Baire-dominated

Baire-dominated

(7) Mařík

The implication $(1) \rightarrow(3)$ follows from [6, 5.2.2]. (2) $\rightarrow(3) \rightarrow(4) \rightarrow(6)$ are obvious. (1) $\rightarrow(7)$ is a classical result of Mařik [18] and is the origin of the name of a Mařik space. (3) $\rightarrow(7)$ was proved by Bachman-Sultan [4], and $(4) \rightarrow(7)$ is a recent result of Adamski [2]. (5) $\rightarrow(7)$ is due to Knowles [15].

Wheeler's questions which we now answer are the following; in his papers [28] and [29], the symbol $(*),((* *))$ was used to denote the property of being (weakly) cozero-dominated.

A [29, Problem 8.12]. Is there a locally compact, MC space which is not paracompact?

B [28, Q6]; [29, Problem 9.10]. Is there an MC space which is not (weakly) cozero-dominated?

C [28, p. 95]. Is Michael's product space (see $\S 2$ below) cozero-dominated or weakly cozero-dominated?

$\mathrm{D}$ [28, Q5]; [29, Problem 9.15]. Is every countably paracompact space a Mařik space?

A perfect map is a closed, continuous map such that the inverse image of each point is compact. A perfect map is called irreducible if it carries a proper closed subset to a proper subset. A space is called extremally disconnected if the closure of every open set is open. Each space $X$ is known to be the image of a unique extremally disconnected space $E(X)$, called the absolute of $X$, under a perfect irreducible map. For details, see [31].

$\mathrm{E}[28, \mathrm{Q} 7]$. Is it true that $X$ is a Mařik space if and only if $E(X)$ is a Mařík space?

The answers to $\mathrm{A}$ and $\mathrm{B}$ are positive, and the answers to $\mathrm{C}, \mathrm{D}$, and $\mathrm{E}$ are negative. 
From now on, $|A|$ denotes the cardinality of a set $A$. As usual, a cardinal is the initial ordinal and an ordinal is the set of smaller ordinals. When viewed as a topological space, a set of ordinals has the order topology. Let $\omega\left(\omega_{1}\right)$ denote the first infinite (uncountable) ordinal, and let $\mathfrak{c}=2^{\omega}$. If $\alpha$ is a cardinal, then the inequality $\alpha<m_{r}$ means that $\alpha$ is not real-valued measurable. For a space $X, Z(X)(\operatorname{Coz}(X))$ is the set of all zero-(cozero-)sets of $X, C(X)$ is the set of all real-valued, continuous functions on $X$, and $C^{*}(X)$ is the set of all bounded functions in $C(X)$. A zero-set of the form $f^{-1}(0)$, where $f \in C(X)$, is denoted by $Z(f)$. The letter $N$ is used for the set of natural numbers.

Our terminology and notation follow [6] and [13]. For recent surveys of Baire measures and of Borel measures, the reader is referred to [29] and [10], respectively.

\section{MC SPACES WHICH ARE NOT BAIRE-DOMINATED}

In this section, we give three examples of MC spaces which are not Bairedominated. They have different features; the first one is locally compact and needs no set theoretic axioms beyond ZFC unlike others; the second one is Michael's product space, which is first countable and submetrizable; and the last one is weakly cozero-dominated. Before proceeding to examples, we show that a nonnormal space yields a space which is not weakly Baire-dominated. Recall that a subspace $S$ of a space $X$ is $C$-embedded in $X$ if every $f \in C(S)$ can be extended continuously over $X$.

Theorem 2.1. For each nonnormal space $X$, there exists a space $Y$ which is not weakly Baire-dominated and which is the countable union of closed, $C$-embedded copies of $X$. Moreover, if $X$ is locally compact, then so is $Y$.
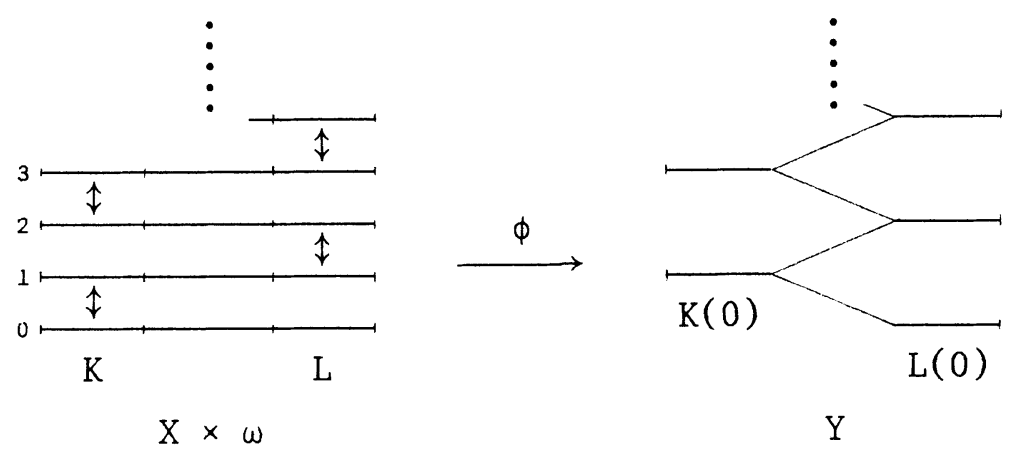

Figure 1

Proof. Let $X$ be a nonnormal space. Then there exists a pair $K, L$ of disjoint closed sets for which every zero-set containing $K$ meets $L$. In fact, take disjoint closed sets $K^{\prime}$ and $L^{\prime}$ which can not be separated by disjoint open sets; then, if there is $Z \in Z(X)$ such that $K^{\prime} \subset Z$ and $Z \cap L^{\prime}=\varnothing$, then the pair $L^{\prime}, Z$ 
is a desired one. Define $Y$ to be the quotient space obtained from the product $X \times \omega$ by identifying points $(k, 2 n)$ with $(k, 2 n+1)$ for each $k \in K$ and $n \in \omega$, and points $(l, 2 n+1)$ with $(l, 2 n+2)$ for each $l \in L$ and $n \in \omega$. Let $\phi: X \times \omega \rightarrow Y$ be the quotient map. For each $n \in \omega$ and each $A \subset X$, let $A(n)=\phi(A \times\{n\})$ (see Figure 1). Then $Y=\bigcup_{n \in \omega} X(n)$, and each $X(n)$ is closed, $C$-embedded in $Y$, and homeomorphic to $X$. To prove that $Y$ is not weakly Baire-dominated, let $\mathscr{F}=\{Z \cap L: K \subset Z \in Z(X)\}$. Then $\mathscr{F}$ is a filter base closed under countable intersections. For each $n \in \omega$ and each $B \subset Y$, let $B\langle n\rangle=p_{n}^{-1}(B \cap L(n))$, where $p_{n}$ is an embedding from $X$ onto $X(n)$. As usual, $S \triangle T=(S-T) \cup(T-S)$.

Claim 1. For each $B \in \mathrm{Ba}(Y)$ and each $0<n<\omega$, there exists $F \in \mathscr{F}$ such that $(B\langle 0\rangle \triangle B\langle n\rangle) \cap F=\varnothing$.

Proof. Since $B\langle 0\rangle \triangle B\langle n\rangle \subset \bigcup_{i<n}(B\langle i\rangle \triangle B\langle i+1\rangle)$, it suffices to prove that for each $i<n$, there exists $F \in \mathscr{F}$ such that $(B\langle i\rangle \triangle B\langle i+1\rangle) \cap F=\varnothing$. In case $i$ is odd, $B\langle i\rangle \triangle B\langle i+1\rangle=\varnothing$, so we prove only the case $i=0$; other even cases are similar. Since

$$
\mathscr{S}=\{S \subset Y:(S\langle 0\rangle \triangle S\langle 1\rangle) \cap F=\varnothing \text { for some } F \in \mathscr{F}\}
$$

is a $\sigma$-algebra, it is sufficient to show that $Z(Y) \subset \mathscr{S}$. To do this, let $Z=$ $Z(f) \in Z(Y)$. Define $f_{i}=f \circ p_{i}$ for $i=0,1$. Since $f_{0}\left|K=f_{1}\right| K$,

$$
K \subset Z\left(f_{0}-f_{1}\right) \text {. }
$$

Thus, if we set $F=Z\left(f_{0}-f_{1}\right) \cap L$, then $F \in \mathscr{F}$. Since $\left(Z\left(f_{0}\right) \triangle Z\left(f_{1}\right)\right) \cap$ $Z\left(f_{0}-f_{1}\right)=\varnothing$ and $Z\langle i\rangle=Z\left(f_{i}\right) \cap L,(Z\langle 0\rangle \triangle Z\langle 1\rangle) \cap F=\varnothing$, and hence $Z \in \mathscr{S}$.

Claim 2. The space $Y$ is not weakly Baire-dominated.

Proof. For each $n \in \omega$, let $D_{n}=\bigcup_{i>n} X(i)$. Then $D_{n}$ is regular closed in $Y$ and $D_{n} \downarrow \varnothing$. Assume that there exist $B_{n} \in \mathrm{Ba}(Y)$ such that $D_{n} \subset B_{n}$ and $B_{n} \downarrow \varnothing$. For each $n \in \omega$, by Claim 1, there exists $F_{n} \in \mathscr{F}$ such that

$$
\left(B_{n}\langle 0\rangle \triangle B_{n}\langle n+1\rangle\right) \cap F_{n}=\varnothing .
$$

Since $L(n+1) \subset D_{n} \subset B_{n}, B_{n}\langle n+1\rangle=L$, so $F_{n} \subset B_{n}\langle 0\rangle$, and hence $F_{n}(0) \subset B_{n} \cap L(0)$. Consequently, $\bigcap_{n \in \omega} B_{n} \supset \bigcap_{n \in \omega} F_{n}(0) \neq \varnothing$, which is a contradiction.

Finally, assume that $X$ is locally compact. Since $\phi$ is a perfect map, it follows from $[6,3.7 .21]$ that $Y$ is then locally compact. Hence the proof is complete.

The following theorem due to Okada-Okazaki [22] shows that, in Theorem 2.1 , if $X$ is MC, then so is $Y$. A subspace $S$ of a space $X$ is said to be Baire-embedded in $X$ if for each $B \in \mathrm{Ba}(S)$, there exists $A \in \mathrm{Ba}(X)$ with $B=A \cap S$. Every $C$-embedded subspace is Baire-embedded (cf. [5, 8.7]). 
Okada-Okazaki's theorem. If $X=\cup_{n \in \omega} X_{n}$, and if each $X_{n}$ is $M C$ and is Baire-embedded in $X$, then $X$ is $M C$.

Remark 2.2. If there exists a nonparacompact MC space $X$, then there exists a nonnormal MC space. Consider the product of $X$ with its Stone-Čech compactification $\beta X$. By [20, Theorem 5.3], $X \times \beta X$ is MC, while it follows from $[6,5.1 .38]$ that it is not normal.

The preceding theorems and remark show that a positive answer to the question A answers B positively. Thus the following example provides answers to both of the questions.

Example 2.3. There exists a locally compact, nonnormal, MC space $X$.

Proof. Let $D$ be a discrete space of cardinality $\omega_{1}$. Consider the product $\beta D \times(\omega+1)$ and its subspace

$$
X=(\beta D \times(\omega+1))-((\beta D-D) \times\{\omega\}) .
$$

Clearly $X$ is locally compact. If we set $K=(\beta D-D) \times \omega$ and $L=D \times\{\omega\}$, then $K$ and $L$ are disjoint closed in $X$ but cannot be separated by disjoint open sets, and hence $X$ is not normal. Define $T=D \times(\omega+1)$; then $X=T \cup K$. It is easily checked that $T$ is MC and is Baire-embedded in $X$. On the other hand, being $\sigma$-compact, $K$ is also MC and is Baire-embedded in $X$ by [5, 9.11]. Hence it follows from Okada-Okazaki's theorem that $X$ is MC.

Remarks 2.4. The space $X$ defined above itself is cozero-dominated. To see this, let $\left\{F_{n}\right\}_{n \in \omega}$ be a sequence of closed sets in $X$ such that $F_{n} \downarrow \varnothing$. Since each $\beta D \times\{m\}, m \in \omega$, is compact, we may assume that $F_{n} \cap(\beta D \times\{m\})=\varnothing$ if $m \leq n$. For each $n \in \omega$, define

$$
G_{n}=F_{n} \cup\left(\bigcup_{n<m<\omega}(\beta D \times\{m\})\right) .
$$

Then $G_{n} \in \operatorname{Coz}(X), F_{n} \subset G_{n}$, and $G_{n} \downarrow \varnothing$. The construction of $X$ was inspired by the argument used by Kato in the proof of his theorem [14, Theorem I].

The second example is Michael's product space $M \times P$. The letters $R, Q$, and $P$ are used to denote the real numbers, rational numbers, and irrational numbers, respectively, and, unless otherwise stated, are assumed to have the usual topologies inherited from $R$. The Michael line $M$ is the set $R$ topologized by isolating the points of $P$ and leaving the points of $Q$ with their usual neighborhoods. As was proved by Michael (cf. [6, 5.1.32]), $M \times P$ is not normal. On the other hand, Moran proved in [20] that $M \times P$ is $\mathrm{MC}$ when c $<m_{r}$. Therefore, by Theorem 2.1, we can make from $M \times P$ an MC space which is not weakly Baire-dominated. Here, in response to the question $C$, we prove the following theorem:

Theorem 2.5. Michael's product space $M \times P$ is not weakly Baire-dominated.

The proof is rather difficult and requires the following two theorems, which may be of some interest in their own right. For a space $X$, let $\mathscr{C}_{1}(X)\left(\mathscr{C}_{2}(X)\right)$ 
denote the family of all subsets of first (second) category in $X$, and define $\mathscr{C}_{0}(X)$ to be the family of all subsets $A$ of $X$ satisfying that every nonempty open set $U$ in $X$ contains a nonempty open subset $V$ such that $V \cap A$ or $V-A$ is in $\mathscr{C}_{1}(X)$. When no confusion can arise, we shall write $\mathscr{C}_{i}$ instead of $\mathscr{C}_{i}(X), i=0,1,2$.

Theorem 2.6. If $X$ is a hereditarily Lindelöf space, then $\mathscr{C}_{0}(X)$ is a $\sigma$-algebra. Proof. Since $V \cap(X-A)=V-A$ and $V-(X-A)=V \cap A, A \in \mathscr{C}_{0}$ implies $X-A \in \mathscr{C}_{0}$. To complete the proof, suppose that $\left\{A_{n}\right\}_{n \in \omega} \subset \mathscr{C}_{0}$ is given, and let $A=\bigcup_{n \in \omega} A_{n}$. To show that $A \in \mathscr{C}_{0}$, fix any nonempty open set $U$ in $X$. We have to prove that there exists a nonempty open set $V \subset U$ such that $V \cap A \in \mathscr{C}_{1}$ or $V-A \in \mathscr{C}_{1}$. If there exists a nonempty open set $V \subset U$ such that $V-A_{n} \in \mathscr{C}_{1}$ for some $n \in \omega$, then $V-A \in \mathscr{C}_{1}$ since $V-A \subset V-A_{n}$. So suppose that for each $n \in \omega$ and each nonempty open set $V \subset U, V-A_{n} \in \mathscr{C}_{2}$. Then, since $A_{n} \in \mathscr{C}_{0}$, each nonempty open set $V \subset U$ has a nonempty open subset $W$ such that $W \cap A_{n} \in \mathscr{C}_{1}$. For each $n \in \omega$, define $\mathscr{W}_{n}$ to be the family of all nonempty open sets $W \subset U$ such that $W \cap A_{n} \in \mathscr{C}_{1}$, and let $W_{n}=\bigcup\left\{W: W \in \mathscr{W}_{n}\right\}$. Then $W_{n}$ is dense in $U$, and hence $U-W_{n} \in \mathscr{C}_{1}$. On the other hand, $X$ being hereditarily Lindelöf, there exists a countable subfamily $\left\{W_{n i}\right\}_{i \in \omega}$ of $\mathscr{W}_{n}$ with $W_{n}=\bigcup_{i \in \omega} W_{n i}$. Since each $W_{n i} \cap A_{n}$ is in $\mathscr{C}_{1}, W_{n} \cap A_{n}=\bigcup_{i \in \omega}\left(W_{n i} \cap A_{n}\right) \in \mathscr{C}_{1}$. Thus $U \cap A_{n}$ is contained in the union of two sets $U-W_{n}$ and $W_{n} \cap A_{n}$ in $\mathscr{C}_{1}$, so $U \cap A_{n} \in \mathscr{C}_{1}$. Consequently, $U \cap A=\bigcup_{n \in \omega}\left(U \cap A_{n}\right) \in \mathscr{C}_{1}$, which completes the proof.

For each $A \subset M \times P$, define $A_{\triangle}=\{x \in P:(x, x) \in A\}$.

Theorem 2.7. If $B \in \mathrm{Ba}(M \times P)$, then $B_{\triangle} \in \mathscr{C}_{0}(P)$.

Proof. By the preceding theorem $\mathscr{C}_{0}(P)$ is a $\sigma$-algebra. Since $\left\{B_{\triangle}: B \in\right.$ $\mathrm{Ba}(M \times P)\}$ is a $\sigma$-algebra generated by a family $\mathscr{G}=\left\{G_{\triangle}: G \in \operatorname{Coz}(M \times P)\right\}$, it suffices to prove that $\mathscr{G} \subset \mathscr{C}_{0}(P)$. Suppose not, and let $G_{\triangle} \in \mathscr{G}-\mathscr{C}_{0}(P)$. Then there exists a nonempty open set $U$ of $P$ such that for each nonempty open set $V \subset U, V \cap G_{\triangle} \in \mathscr{C}_{2}$ and $V-G_{\triangle} \in \mathscr{C}_{2}$. Since $G$ is a cozero-set, there exists an increasing sequence $\left\{G_{i}\right\}_{i \in \omega} \subset \operatorname{Coz}(M \times P)$ such that $\mathrm{cl}_{M \times P} G_{i} \subset G_{i+1}$, $i \in \omega$, and $G=\bigcup_{i \in \omega} G_{i}$. For each $i, j \in \omega$, define

$$
A_{i j}=\left\{x \in U \cap G_{\triangle}:\{x\} \times B_{j}(x) \subset G_{i}\right\},
$$

where $B_{j}(x)=\left\{y \in P:|x-y|<1 / 2^{j}\right\}$. If $x \in U \cap G_{\triangle}$, then, since $(x, x) \in G$, $\{x\} \times B_{j}(x) \subset G_{i}$ for some $i, j \in \omega$, so $x \in A_{i j}$. Hence $U \cap G_{\triangle}=\bigcup_{i, j \in \omega} A_{i j}$. By the hypothesis of $U, U \cap G_{\triangle} \in \mathscr{C}_{2}$, so there exist $k, l \in \omega$ such that $A_{k l} \in \mathscr{C}_{2}$. Therefore, there exists a nonempty open set $V \subset U$ such that

$$
V \cap A_{k l} \text { is dense in } V .
$$

Fix such $k, l, V$, and define

$$
B_{m}=\left\{x \in V-G_{\triangle}:\left(\{x\} \times B_{m}(x)\right) \cap G_{k+1}=\varnothing\right\}
$$


for each $m \in \omega$. If $x \in V-G_{\triangle}$, then, since $(x, x) \notin G,\left(\{x\} \times B_{m}(X)\right) \cap$ $G_{k+1}=\varnothing$ for some $m \in \omega$, so $x \in B_{m}$. Hence $V-G_{\triangle}=\bigcup_{m \in \omega} B_{m}$. By the hypothesis of $U$ again, $V-G_{\triangle} \in \mathscr{C}_{2}$, so there exist $n \in \omega$ and a nonempty open set $W \subset V$ such that

$$
W \cap B_{n} \text { is dense in } W .
$$

Define $s=\max \{l, n\}$, and pick a point $q \in\left(\operatorname{cl}_{R} W\right) \cap Q$. Then, by (1) and (2), $q \in\left(\mathrm{cl}_{R} A_{k l}\right) \cap\left(\mathrm{cl}_{R} B_{n}\right)$. Since $q \in \mathrm{cl}_{R} A_{k l}$,

$$
\{q\} \times B_{s}(q) \subset \mathrm{cl}_{M \times P}\left(\bigcup\left\{\{x\} \times B_{s}(x): x \in A_{k l}\right\}\right) \subset \mathrm{cl}_{M \times P} G_{k} .
$$

On the other hand, since $q \in \mathrm{cl}_{R} B_{n}$,

$$
\{q\} \times B_{s}(q) \subset \operatorname{cl}_{M \times P}\left(\bigcup\left\{\{x\} \times B_{s}(x): x \in B_{n}\right\}\right) \subset(M \times P)-G_{k+1} .
$$

This contradicts the fact that $\mathrm{cl}_{M \times P} G_{k} \subset G_{k+1}$. Hence $\mathscr{G} \subset \mathscr{C}_{0}(P)$, which completes the proof.

Corollary 2.8. If $A \in \mathrm{Ba}(M)$, then $A \cap P \in \mathscr{C}_{0}(P)$.

Proof. Since $A \in \mathrm{Ba}(M), B=A \times P \in \mathrm{Ba}(M \times P)$. Hence it follows from Theorem 2.7 that $B_{\triangle}=A \cap P \in \mathscr{C}_{0}(P)$.

Proof of Theorem 2.5.

Claim 1. There exists a sequence $\left\{X_{n}\right\}_{n \in \omega}$ of subsets of $P$ such that $X_{n} \downarrow \varnothing$ and for each $n \in \omega$ and each nonempty open set $U$ of $P, U \cap X_{n} \in \mathscr{C}_{2}(P)$.

Proof. By Bernstein's theorem (cf. $[15, \S 40]$ ), there exists a partition $\left\{Y_{n}\right\}_{n \in \omega}$ of $P$ such that $\left|K \cap Y_{n}\right|=\mathfrak{c}$ for each $n \in \omega$ and each uncountable closed set $K$ of $P$. Define $X_{n}=\bigcup_{k \geq n} Y_{k}, n \in \omega$. Then, obviously $X_{n} \downarrow \varnothing$. For our end, let $n \in \omega$ and let $U$ be a nonempty open set of $P$. If $U \cap X_{n} \in \mathscr{C}_{1}$, then $U \cap Y_{n} \in \mathscr{C}_{1}$ since $Y_{n} \subset X_{n}$, so there exists a sequence $\left\{D_{i}\right\}_{i \in \omega}$ of nowhere dense closed subsets of $P$ with $U \cap Y_{n} \subset \bigcup_{i \in \omega} D_{i}$. Let $E=U-\bigcup_{i \in \omega} D_{i}$. Since $U$ is nonempty open, $U \in \mathscr{C}_{2}$, and hence so is $E$. Therefore $E$ is an uncountable Borel set of $P$. Hence it follows from [15, $\S 37$, Theorem 3] that $E$ contains a copy of the Cantor set $C$. By the property of $Y_{n},\left|C \cap Y_{n}\right|=\mathfrak{c}$, while

a contradiction.

$$
C \cap Y_{n} \subset\left(U \cap Y_{n}\right)-\bigcup_{i \in \omega} D_{i} \subset\left(\bigcup_{i \in \omega} D_{i}\right)-\left(\bigcup_{i \in \omega} D_{i}\right)=\varnothing,
$$

For each $n \in \omega$, define

$$
F_{n}=\mathrm{cl}_{M \times P}\left(\bigcup\left\{\{x\} \times B_{n}(x): x \in X_{n}\right\}\right),
$$

where $B_{n}(x)$ is the same as in the proof of Theorem 2.7. Then each $F_{n}$ is regular closed in $M \times P$, and it is easily checked that $F_{n} \downarrow \varnothing$. To show that $M \times P$ is not weakly Baire-dominated, let $\left\{B_{n}\right\}_{n \in \omega}$ be a sequence in $\mathrm{Ba}(M \times P)$ such that $F_{n} \subset B_{n}$ for each $n \in \omega$. We have to prove that $\bigcap_{n \in \omega} B_{n} \neq \varnothing$. 
Claim 2. Suppose that $X$ is a subset of $P$ such that for each nonempty open set $U$ of $P, U \cap X \in \mathscr{C}_{2}(P)$, and $B$ is a Baire set of $M \times P$ containing $\{(x, x): x \in X\}$. Then $P-B_{\triangle} \in \mathscr{C}_{1}(P)$.

Proof. By Theorem 2.7, $B_{\triangle} \in \mathscr{C}_{0}(P)$. Since $X \subset B_{\triangle}, U \cap B_{\triangle} \in \mathscr{C}_{2}$ for each nonempty open set $U$ of $P$. Hence, by the definition of $\mathscr{C}_{0}(P)$, for each nonempty open set $U$ of $P$, there exists a nonempty open set $V \subset U$ such that $V-B_{\triangle} \in \mathscr{C}_{1}$. Define $\mathscr{V}$ to be the family of all nonempty open sets $V$ of $P$ such that $V-B_{\triangle} \in \mathscr{C}_{1}$, and let $W=\bigcup\{V: V \in \mathscr{V}\}$. Then $W$ is open and dense in $P$, so $P-W$ is nowhere dense in $P$. On the other hand, $P$ being hereditarily Lindelöf, there exists a countable subfamily $\left\{V_{i}\right\}_{i \in \omega}$ of $\mathscr{V}$ with $W=\bigcup_{i \in \omega} V_{i}$. Since $V_{i}-B_{\triangle} \in \mathscr{C}_{1}, W-B_{\triangle}=\bigcup_{i \in \omega}\left(V_{i}-B_{\triangle}\right) \in \mathscr{C}_{1}$. Since $P-B_{\triangle} \subset(P-W) \cup\left(W-B_{\triangle}\right), P-B_{\triangle} \in \mathscr{C}_{1}$, thus proving the claim.

Since $\left\{(x, x): x \in X_{n}\right\} \subset F_{n} \subset B_{n}$, it follows from Claim 2 that $P-\left(B_{n}\right)_{\triangle} \in$ $\mathscr{C}_{1}$. Thus $\left(\bigcap_{n \in \omega} B_{n}\right)_{\triangle}=\bigcap_{n \in \omega}\left(B_{n}\right)_{\triangle} \neq \varnothing$, and hence $\bigcap_{n \in \omega} B_{n} \neq \varnothing$. The proof of Theorem 2.5 is now completed.

The following corollary (to the proof of Theorem 2.5) will be used in $\S 4$.

Corollary 2.9. Suppose that $X$ is a subset of $P$ such that for each nonempty open set $U$ of $P, U \cap X \in \mathscr{C}_{2}(P)$, and $X \subset A \in \mathrm{Ba}(M)$. Then $P-A \in \mathscr{C}_{1}(P)$. Proof. Apply Claim 2 in the proof of Theorem 2.5 by putting $B=A \times P$. Then $P-B_{\triangle}=P-(A \cap P)=P-A \in \mathscr{C}_{1}(P)$.

The third example needs Martin's axiom plus the negation of the continuum hypothesis, abbreviated as $\mathrm{MA}+\neg \mathrm{CH}$, from which $\mathfrak{c}<m_{r}$ is deduced. Under this assumption, there exist many examples of nonparacompact $\mathrm{MC}$ spaces. For example, every nonmetrizable, normal, Moore space of cardinality $\leq \mathfrak{c}$ is this case (cf. [25, §IV]); however, such a space is countably paracompact by itself. Perhaps the most interesting one is the nonnormal space $N^{\omega_{1}}$, the product of $\omega_{1}$ many copies of a countable discrete space $N$. It was proved by Fremlin in [7] that $N^{\omega_{1}}$ is MC under MA $+\neg \mathrm{CH}$. The space $N^{\omega_{1}}$ has the following properties:

Theorem 2.10. For each $\lambda>\omega, N^{\lambda}$ is weakly cozero-dominated but not Bairedominated.

Proof. If $B$ is a regular closed set or a Baire set of $N^{\lambda}$, then, by [23, Theorem 3] and [24, Theorem 2.3], it is a set of the form $\pi_{\Lambda}^{-1}\left(\pi_{\Lambda}(B)\right)$ for some countable subset $\Lambda$ of $\lambda$, where $\pi_{\Lambda}$ is the projection from $N^{\lambda}$ to $N^{\Lambda}$. Hence the first assertion is easily proved. To prove that $N^{\lambda}$ is not Baire-dominated, let

$$
F_{n}=\left\{f \in N^{\lambda}: \text { for each } i \leq n,|\{\alpha \in \lambda: f(\alpha)=i\}| \leq 1\right\}
$$

for each $n \in N$. Then $F_{n}$ is closed in $N^{\lambda}$ and $F_{n} \downarrow \varnothing$. Assume that there exists a sequence $\left\{B_{n}\right\}_{n \in N} \subset \mathrm{Ba}\left(N^{\lambda}\right)$ such that $F_{n} \subset B_{n}$ and $B_{n} \downarrow \varnothing$. Then there exists a countable subset $M$ of $\lambda$ such that for each $n \in N, B_{n}=$ 
$\pi_{M}^{-1}\left(\pi_{M}\left(B_{n}\right)\right)$. If we choose a bijection $g: M \rightarrow N$, then $g \in \bigcap_{n \in N} \pi_{M}\left(F_{n}\right) \subset$ $\bigcap_{n \in N} \pi_{M}\left(B_{n}\right)$, and hence $\bigcap_{n \in N} B_{n} \neq \varnothing$, which is a contradiction.

We have been unable to decide if $N^{\omega_{1}}$, or more generally $N^{\lambda}$, is a Mařík space without MA $+\neg \mathrm{CH}$. If regularity of the Borel extension is negligible, then we have a much stronger result. To show this, we call a space $X$ a quasi-Mařik space if each Baire measure on $X$ admits an extension to a (not necessarily regular) Borel measure on $X$. For a space $X, v X$ denotes the Hewitt realcompactification of $X$.

Theorem 2.11. Assume that $X$ is a quasi-Mařik space and $X \subset Y \subset v X$. Then $Y$ is a quasi-Mařik space.

Proof. Let $\mu$ be a Baire measure on $Y$. As is well known, every $B \in \mathrm{Ba}(X)$ extends to a unique $B^{v} \in \mathrm{Ba}(v X)$ in such a manner that if $\left\{B_{n}\right\}_{n \in \omega}$ is disjoint, then so is $\left\{B_{n}^{v}\right\}_{n \in \omega}$. Therefore, if we define $\mu_{X}(B)=\mu\left(B^{v} \cap Y\right)$ for $B \in \mathrm{Ba}(X)$, then $\mu_{X}$ is a Baire measure on $X$. By the assumption, $\mu_{X}$ extends to a Borel measure $\nu_{X}$ on $X$. For each $A \in \operatorname{Bo}(Y)$, define $\nu(A)=\nu_{X}(A \cap X)$. Then $\nu$ is a Borel extension of $\mu$.

Corollary 2.12. For any family $\left\{X_{\alpha}\right\}_{\alpha \in \lambda}$ of metric spaces, the product $X=$ $\Pi_{c \in i} X_{c}$ is a quasi-Mařik space. In particular, $N^{\lambda}$ is a quasi-Mařik space.

Proof. Consider a $\Sigma$-product $\Sigma$ of $X$; i.e., define

$$
\Sigma=\{f \in X:|\{\alpha \in \lambda: f(\alpha) \neq g(\alpha)\}| \leq \omega\} \subset X,
$$

where $g$ is a fixed point of $X$. By [26, Theorem 2.2] $\Sigma$ is $C$-embedded in $X$, so $X \subset v \Sigma$. By [12, Theorem 1] $\Sigma$ is normal, and by [6,5.2.9] $\Sigma$ is countably paracompact. Consequently, $\Sigma$ is a Maŕik space, and hence it follows from Theorem 2.11 that $X$ is a quasi-Maŕík space.

The following questions remain open.

Question 2.13. Is $N^{\lambda}$ a Mařík space for each cardinal $\lambda$ ?

Question 2.14. Is $v X$ a Mařík space, whenever $X$ is?

Question 2.15. Is every quasi-Mařík space a Mařík space?

By our results, a positive answer to 2.15 answers 2.14 positively, and a positive answer to 2.14 answers 2.13 positively.

\section{NON-MAŘ́́K SPACES}

In this section, we answer both of the questions $\mathrm{D}$ and $\mathrm{E}$ negatively. Let us begin by making criteria to check that a space is not a quasi-Mařík space. Some terms and symbols are needed. The continuous extension of $f \in C^{*}(X)$ over $\beta X$ is denoted by $f^{\beta}$. Clearly, $Z(f)=Z\left(f^{\beta}\right) \cap X$. A measure is called locally zero if each point has a neighborhood of measure zero. Let $\mu$ be a Baire measure on $X$; then $\mu^{\beta}$ denotes the Baire measure on $\beta X$ defined by 
$\mu^{\beta}(B)=\mu(B \cap X)$ for $B \in \operatorname{Ba}(\beta X)$. Define $S\left(\mu^{\beta}\right)=\bigcap\left\{Z \in Z(\beta X): \mu^{\beta}(Z)=\right.$ $\left.\mu^{\beta}(\beta X)\right\}$, which is called the support of $\mu^{\beta}$. A space $X$ is called a $D$-space if for each discrete subspace $S \subset X,|S|<\mathfrak{m}_{r}$ (cf. [29]).

Theorem 3.1. Let $\mu$ be a locally zero, Baire measure on a space $X$ with $\mu(X)>$ 0 . Assume that there exist $Z_{0}=Z(f) \in Z(X)$ such that $S\left(\mu^{\beta}\right) \subset Z\left(f^{\beta}\right), a$ continuous map $\psi$ from $Z_{0}$ to a paracompact $D$-space $Y$, and an open cover $\mathscr{U}$ of $Y$ satisfying the following condition: (1) For each $U \in \mathscr{U}$, there exists $B \in \mathrm{Ba}(X)$ such that $\psi^{-1}(U) \subset B$ and $\mu(B)=0$. Then $\mu$ cannot be extended to any Borel measure on $X$.

Proof. Let $\mathscr{S}=\left\{Z \in Z(\beta X): \mu^{\beta}(Z)=\mu^{\beta}(\beta X)\right\}$. Then $Z_{1}, Z_{2} \in \mathscr{S}$ implies that $Z_{1} \cap Z_{2} \in \mathscr{S}$. Thus, $\left\{Z\left(f^{\beta}\right) \cup Z: Z \in \mathscr{S}\right\}$ is a net. Since $S\left(\mu^{\beta}\right) \subset Z\left(f^{\beta}\right)$,

$$
Z\left(f^{\beta}\right)=\bigcap\left\{Z\left(f^{\beta}\right) \cup Z: Z \in \mathscr{S}\right\} .
$$

Since $\beta X$ is MC, $\mu^{\beta}$ is $\tau$-additive. This can be combined with (2) to yield that

$$
\left.\mu^{\beta}\left(Z\left(f^{\beta}\right)\right)=\inf \left\{\mu^{\beta}\left(Z\left(f^{\beta}\right) \cup Z\right)\right\}: Z \in \mathscr{S}\right\}=\mu^{\beta}(\beta X) .
$$

Hence, $\mu\left(Z_{0}\right)=\mu^{\beta}\left(Z\left(f^{\beta}\right)\right)=\mu^{\beta}(\beta X)=\mu(X)>0$. Suppose that there exists a Borel extension $\nu$ of $\mu$. For each $A \in \operatorname{Bo}(Y)$, define $\nu_{Y}(A)=\nu\left(\psi^{-1}(A)\right)$. By condition (1), $\nu_{Y}$ is a locally zero, Borel measure on $Y$. It is known [10, 7.6 and 10.2] that every locally zero, Borel measure on a paracompact $D$-space is identically zero. Hence $\nu_{Y}(Y)=0$, while $\nu_{Y}(Y)=\nu\left(Z_{0}\right)=\mu\left(Z_{0}\right)>0$, which is a contradiction.

Recall from [11,6.5 and 8.7] that for each $p \in \nu X, A^{p}=\{Z \in Z(X)$ : $\left.p \in \mathrm{cl}_{\beta X} Z\right\}$ is an ultrafilter in $Z(X)$ with the countable intersection property. Define a map $\mu_{p}: \mathrm{Ba}(X) \rightarrow\{0,1\}$ by $\mu_{p}(B)=1$ if $B$ includes some element of $A^{p}$, and $\mu_{p}(B)=0$ otherwise. Then $\mu_{p}$ is a Baire measure on $X$ such that $S\left(\mu_{p}^{\beta}\right)=\{p\} \quad$ (cf. also $[10,8.11]$ ).

Corollary 3.2. Assume that there exist $p \in v X-X, Z_{0} \in Z(X)$ with $p \in$ $\mathrm{cl}_{\beta X} Z_{0}$, and a closed, continuous map $\psi$ from $Z_{0}$ to a paracompact $D$-space $Y$ such that $p \notin \operatorname{cl}_{\beta X} \psi^{-1}(y)$ for each $y \in Y$. Then $X$ is not a quasi-Mařik space.

Proof. Let $\mu_{p}$ be the $\{0,1\}$-valued Baire measure on $X$ defined as above. Then $S\left(\mu_{p}^{\beta}\right)=\{p\} \subset Z\left(f^{\beta}\right)$ by our assumption. For each $y \in Y$, choose $V_{y} \in \operatorname{Coz}(\beta X)$ such that $\operatorname{cl}_{\beta X} \psi^{-1}(y) \subset V_{y}$ and $p \notin \operatorname{cl}_{\beta X} V_{y}$. Then, since $p \in \mathrm{cl}_{\beta X}\left(X-V_{y}\right), \mu_{p}\left(X-V_{y}\right)=1$, and hence $\mu_{p}\left(X \cap V_{y}\right)=0$. Since $\psi$ is closed, there exists an open neighborhood $U_{y}$ of $y$ in $Y$ such that $\psi^{-1}\left(U_{y}\right) \subset V_{y}$. Put $\mathscr{U}=\left\{U_{y}: y \in Y\right\}$; then $\mathscr{U}$ satisfies the condition (1) in the preceding theorem. Hence, $\mu_{p}$ cannot be extended to any Borel measure on $X$. 
Corollary 3.3. Assume that there exists $Z_{0} \in Z(X)$, which is a paracompact $D$-space as a subspace, such that $\mathrm{cl}_{\beta X} Z_{0} \cap(v X-X) \neq \varnothing$. Then $X$ is not a quasi-Mařik space.

Proof. This follows from Corollary 3.2 if we consider the identity of $Z_{0}$ as $\psi$.

Remarks 3.4. (1) In 3.1, 3.2, and 3.3, "paracompact $D$-space" can be weakened to a space on which each locally zero, Borel measure is identically zero. Such a space was investigated by Gardner [9] and Adamski [1] and is now called a weakly Borel measure complete space. It is known that every weakly $\theta$-refinable $D$-space is weakly Borel measure complete. For details, see [10].

(2) Two typical examples of non-Mařik spaces were exhibited by Wheeler in [28]; the square $S^{2}$ of the Sorgenfrey lines under $\mathfrak{c}<\mathfrak{m}_{r}$ and the Dieudonne Plank D. We can reconfirm that those spaces are not quasi-Mařík spaces by use of Theorem 3.1 and Corollary 3.3, respectively.

The following example, which is a cubic deformation of the Dieudonne Plank, provides a negative answer to the question $\mathrm{D}$.

Example 3.5. There exists a countably paracompact space $X$ which is not a quasi-Mařík space.

Proof. Step I. Let $\omega_{2}$ be the second, uncountable, initial ordinal. Note that $\omega_{2} \neq \omega^{2} ; \omega^{2}$ denotes the square of $\omega$. Define

$$
\begin{gathered}
A=\left(\left(\omega_{2}+1\right) \times\left(\omega_{2}+1\right)\right)-\left\{\left(\omega_{2}, \omega_{2}\right)\right\} \quad \text { and } \\
G_{A}(\alpha)=\left\{\left(\alpha_{1}, \alpha_{2}\right) \in A: \alpha<\alpha_{i} \leq \omega_{2}, i=1,2\right\}
\end{gathered}
$$

for $\alpha \in \omega_{2}$. Then $A$ is $\omega_{1}$-compact, i.e., every open cover of cardinality $\leq \omega_{1}$ has a finite subcover, and it is easily checked that each $f \in C(A)$ is constant on some $G_{A}(\alpha)$. Let us set $A_{\square}=A \times \omega^{2}$. For each $\beta \in \omega^{2}$, there exist uniquely $n, m \in \omega$ and $i \in\{0,1\}$ such that $\beta=\omega n+2 m+i$. Let $B$ be the quotient space obtained from $A_{\square}$ by identifying points $\left(\omega_{2}, \alpha, \omega n+2 m\right)$ with $\left(\omega_{2}, \alpha, \omega n+2 m+1\right)$ for each $\alpha \in \omega_{2}$ and $n, m \in \omega$, and points $\left(\alpha, \omega_{2}, \omega n+\right.$ $2 m+1)$ with $\left(\alpha, \omega_{2}, \omega n+2 m+2\right)$ for each $\alpha \in \omega_{2}$ and $n, m \in \omega$. Then the quotient map $\phi: A_{\square} \rightarrow B$ is perfect. For each $\alpha \in \omega_{2}$ and each $n \in \omega$, let

$$
\begin{aligned}
& G(\alpha)=\phi\left(G_{A}(\alpha) \times \omega^{2}\right) \text { and } \\
& B(n)=\phi\left(A \times\left\{\beta \in \omega^{2}: \omega n \leq \beta<\omega^{2}\right\}\right) .
\end{aligned}
$$

In what follows, a subspace $S$ of a space $T$ is said to be normally placed in $T$ if for every open set $U$ with $S \subset U$, there exists an open set $V$ such that $S \subset V \subset \mathrm{cl}_{T} V \subset U$.

Claim 1. For each $n \in \omega, B(n)$ is closed and normally placed in $B, B(n+1) \subset$ int $_{B} B(n)$, and $\bigcap_{n \in \omega} B(n)=\varnothing$.

Proof. Let $U$ be an open set with $B(n) \subset U$, and let

$$
\beta_{*}=\sup \left(\pi\left[A_{\square}-\phi^{-1}(U)\right]\right),
$$


where $\pi$ is the projection from $A_{\square}$ to $\omega^{2}$. Since $\pi$ is closed by [6, 3.7.10], $\beta_{*}<\omega n$. Define

$$
V=\phi\left[A \times\left\{\beta \in \omega^{2}: \beta_{*}+2 \leq \beta<\omega^{2}\right\}\right] .
$$

Then $V$ is closed in $B$ and $B(n) \subset$ int $_{B} V \subset V \subset U$, and hence $B(n)$ is normally placed in $B$. Other assertions are obvious.

Claim 2. Each $f \in C(B)$ is constant on some $G(\alpha)$.

Proof. For each $\beta \in \omega^{2}$, there exists $\alpha_{\beta} \in \omega_{2}$ such that $f \circ \phi$ is constant on $G_{A}\left(\alpha_{\beta}\right) \times\{\beta\}$. Let $\alpha=\sup \left\{\alpha_{\beta}: \beta \in \omega^{2}\right\}$. Then $\alpha<\omega_{2}$ and $f$ is constant on $G(\alpha)$.

Step II. Define $C=B \times(\omega+1)$ and $C_{\nabla}=\bigcup_{n \in \omega}(B(n) \times\{n\}) \subset C$.

Claim 3. The space $C$ is $\omega_{1}$-paracompact; i.e., every open cover of cardinality $\leq \omega_{1}$ has a locally finite, open refinement.

Proof. In [17] Mack proved that the product of an $\omega_{1}$-compact space with a metric space is $\omega_{1}$-paracompact. Hence $A_{\square} \times(\omega+1)=A \times\left(\omega^{2} \times(\omega+1)\right)$ is $\omega_{1}$ paracompact. If id is the identity of $\omega+1$, then $\phi \times$ id is a perfect map from $A_{\square} \times(\omega+1)$ onto $C$. By [17, Theorem 16] again, $C$ is $\omega_{1}$-paracompact.

Claim 4. The set $C_{\nabla}$ is closed and normally placed in $C$.

Proof. Since $\bigcap_{n \in \omega} B(n)=\varnothing, C_{\nabla}$ is closed in $C$. Let $U$ be an open set with $C_{\nabla} \subset U$, and let $\pi_{B}: C \rightarrow B$ denote the projection. For each $n \in \omega$, since $B(n)$ is normally placed in $B$ by Claim 1 , there exists an open set $V_{n}$ in $B$ such that

$$
B(n) \subset V_{n} \subset \mathrm{cl}_{B} V_{n} \subset \operatorname{int}_{B} B(n-1) \cap \pi_{B}[U \cap(B \times\{n\})],
$$

where $B(-1)=B$. Define $V=\bigcup_{n \in \omega}\left(V_{n} \times\{n\}\right)$. Then $V$ is open in $C$ and $C_{\nabla} \subset V$. Since $\bigcap_{n \in \omega} \mathrm{cl}_{B} V_{n} \subset \bigcap_{n \in \omega} B(n-1)=\varnothing, \mathrm{cl}_{C} V \subset U$.

Step III. Let $D$ be the set $\omega_{1}+1$ with the topology obtained from the order topology by making all points of $\omega_{1}$ isolated. Define

$$
X=(C \times D)-\left(\left(C-C_{\nabla}\right) \times\left\{\omega_{1}\right\}\right),
$$

topologized as a subspace of $C \times D$. We show that $X$ is the desired example. Let $D_{0}=D-\left\{\omega_{1}\right\}$.

Claim 5. The space $X$ is countably paracompact.

Proof. Let $\left\{F_{n}\right\}_{n \in \omega}$ be a sequence of closed sets in $X$ with $F_{n} \downarrow \varnothing$. By $[6,5.2 .1]$ it suffices to find open sets $W_{n}$ in $X$ such that $F_{n} \subset W_{n}$ and $\mathrm{cl}_{X} W_{n} \downarrow \varnothing$. In case $\bigcap_{n \in \omega} \mathrm{cl}_{C \times D} F_{n} \neq \varnothing$, there exists a closed set $F \subset C$ with $\bigcap_{n \in \omega}{ }^{\mathrm{cl}}{ }_{C \times D} F_{n}=F \times\left\{\omega_{1}\right\}$. Since $F \cap C_{\nabla}=\varnothing$, by Claim 4 there exists an open set $H \subset C$ such that $F \subset H \subset \mathrm{cl}_{C} H \subset C-C_{\nabla}$. Let $E=\mathrm{cl}_{C} H \times D_{0}$. Since $\mathrm{cl}_{C} H$ is countably paracompact by Claim 3 and $D_{0}$ is discrete, $E$ is countably paracompact. Thus there exist open sets $U_{n}^{\prime}$ in $E$ such that $F_{n} \cap E \subset U_{n}^{\prime}$ and 
$\mathrm{cl}_{E} U_{n}^{\prime} \downarrow \varnothing$. For each $n \in \omega$, define $U_{n}=U_{n}^{\prime} \cap\left(H \times D_{0}\right)$. Then $U_{n}$ is open in $X$ and

$$
F_{n} \cap\left(H \times D_{0}\right) \subset U_{n} \text { and } \operatorname{cl}_{X} U_{n} \downarrow \varnothing .
$$

In case $\bigcap_{n \in \omega} \mathrm{cl}_{C \times D} F_{n}=\varnothing$, define $U_{n}=H=\varnothing, n \in \omega$. Observe that $U_{n}$ and $H$ then also satisfy (1). Next, for each $n \in \omega$, let $F_{n}^{\prime}=\operatorname{cl}_{C \times D} F_{n}-(H \times D)$. Then $F_{n}^{\prime}$ is closed in $C \times D$ and $F_{n}^{\prime} \downarrow \varnothing$. We show that there exists a sequence $\left\{V_{n}\right\}_{n \in \omega}$ of open sets in $C \times D$ satisfying that

$$
F_{n}^{\prime} \subset V_{n} \text { and } \operatorname{cl}_{C \times D} V_{n} \downarrow \varnothing \text {. }
$$

Since $C$ is countably paracompact, there exist open sets $I_{n} \subset C$ such that

$$
F_{n}^{\prime} \cap\left(C \times\left\{\omega_{1}\right\}\right) \subset I_{n} \times\left\{\omega_{1}\right\} \text { and } \operatorname{cl}_{C} I_{n} \downarrow \varnothing .
$$

Define $F^{\prime}=\bigcup_{n \in \omega}\left(F_{n}^{\prime}-\left(I_{n} \times D\right)\right)$. Then $F^{\prime}$ is closed in $C \times D$ and $F^{\prime} \cap(C \times$ $\left.\left\{\omega_{1}\right\}\right)=\varnothing$. For each $\xi \in D_{0}$, let $J_{\xi}$ be the union of all open sets $G$ in $C$ such that $\left(G \times\left(\xi, \omega_{1}\right]\right) \cap F^{\prime}=\varnothing$, where $\left(\xi, \omega_{1}\right]=\left\{\eta \in D: \xi<\eta \leq \omega_{1}\right\}$. Then $\mathscr{J}=$ $\left\{J_{\xi}: \xi \in D_{0}\right\}$ is an open cover of $C$ such that $J_{\xi} \subset J_{\xi^{\prime}}$ if $\xi<\xi^{\prime}$. By Claim 3 and [17, Theorem 5], there exists a locally finite open refinement $\left\{K_{\xi}: \xi \in D_{0}\right\}$ of $\mathscr{J}$ such that $\mathrm{cl}_{C} K_{\xi} \subset J_{\xi}$ for each $\xi \in D_{0}$. Let $L=\bigcup_{\xi \in D_{0}}\left(K_{\xi} \times\left(\xi, \omega_{1}\right]\right)$. Then $L$ is an open set in $C \times D$ such that

$$
C \times\left\{\omega_{1}\right\} \subset L \subset \mathrm{cl}_{C \times D} L \subset(C \times D)-F^{\prime} .
$$

Since $C \times D_{0}$ is countably paracompact, there exist open sets $O_{n} \subset C \times D_{0}$ such that

$$
F_{n}^{\prime} \cap\left(C \times D_{0}\right) \subset O_{n} \text { and } \operatorname{cl}_{C \times D_{0}} O_{n} \downarrow \varnothing .
$$

For each $n \in \omega$, let $V_{n}=\left(I_{n} \times D\right) \cup\left(O_{n}-\mathrm{cl}_{C \times D} L\right)$. Then $V_{n}$ 's are open in $C \times D$ and satisfy (2). Finally, define $W_{n}=U_{n} \cup\left(V_{n} \cap X\right)$ for each $n \in \omega$. Then $W_{n}$ is open in $X$, and it follows from (1) and (2) that $F_{n} \subset W_{n}$ and $\mathrm{cl}_{X} W_{n} \downarrow \varnothing$, as required.

Recall that $X \subset B \times(\omega+1) \times D$. In the rest, we denote a point of $X$ by a triplet, such as $(b, m, \eta)$, of points of $B, \omega+1$, and $D$. For each $\alpha \in \omega_{2}$, $n \in \omega$, and $\xi \in D_{0}$, let

$$
G(\alpha, n, \xi)=\left\{(b, m, \eta) \in X: b \in G(\alpha), n<m \leq \omega, \xi<\eta \leq \omega_{1}\right\} .
$$

We add an ideal point $x_{\infty}$ to $X$, and define a neighborhood base of $x_{\infty}$ by $\left\{\left\{x_{\infty}\right\} \cup G(\alpha, n, \xi): \alpha \in \omega_{2}, n \in \omega, \xi \in D_{0}\right\}$.

Claim 6. The point $x_{\infty}$ is in $v X$.

Proof. By [5, 1.16], it suffices to prove that $X$ is $C$-embedded in $X \cup\left\{x_{\infty}\right\}$. To do this, let $f \in C(X)$. For each $n \leq \omega$ and each $\xi \in D_{0}$, by Claim 2 there exists $\alpha_{n \xi} \in \omega_{2}$ such that $f$ takes on the constant value $r_{n \xi}$ on $\{(b, n, \xi): b \in$ $\left.G\left(\alpha_{n \xi}\right)\right\}$. Let $\alpha_{*}=\sup \left\{\alpha_{n \xi}: n \leq \omega, \xi \in D_{0}\right\}$; then $\alpha_{*}<\omega_{2}$. For each $n \in \omega$, pick $b_{n} \in G\left(\alpha_{*}\right) \cap B(n)$. Since each $G_{\delta}$ in $D$ is open, there exists $\xi_{n} \in D_{0}$ such 
that $f$ is constant on $\left\{\left(b_{n}, n, \eta\right): \xi_{n}<\eta \leq \omega_{1}\right\}$. Let $\xi_{*}=\sup \left\{\xi_{n}: n \in \omega\right\}$. Then, for each $n \in \omega, \xi_{*}<\xi<\xi^{\prime}<\omega_{1}$ imply that $r_{n \xi}=r_{n \xi^{\prime}}$. We denote this constant value by $r_{n}$. Pick $b^{\prime} \in G\left(\alpha_{*}\right)$. Then, for each $\xi$ with $\xi_{*}<\xi<\omega_{1}$,

$$
\begin{aligned}
r_{\omega \xi} & =f\left(\left(b^{\prime}, \omega, \xi\right)\right)=f\left(\lim _{n \rightarrow \infty}\left(b^{\prime}, n, \xi\right)\right) \\
& =\lim _{n \rightarrow \infty} f\left(\left(b^{\prime}, n, \xi\right)\right)=\lim _{n \rightarrow \infty} r_{n},
\end{aligned}
$$

so define $r_{\omega}=\lim _{n \rightarrow \infty} r_{n}$. Then $f((b, n, \eta))=r_{n}$ whenever $b \in G\left(\alpha_{*}\right)$, $n \leq \omega$, and $\xi_{*}<\eta \leq \omega_{1}$. Extend $f$ over $X \cup\left\{x_{\infty}\right\}$ by setting $f\left(x_{\infty}\right)=r_{\omega}$. To check that $f$ is continuous at $x_{\infty}$, let $\varepsilon>0$. Then there exists $n_{0} \in \omega$ such that if $n>n_{0},\left|r_{n}-r_{\omega}\right|<\varepsilon$. This implies that if $x \in G\left(\alpha_{*}, n_{0}, \xi_{*}\right)$, then $\left|f(x)-f\left(x_{\infty}\right)\right|<\varepsilon$. Hence $f$ can be extended continuously to $x_{\infty}$.

Claim 7. The space $X$ is not a quasi-Maŕík space.

Proof. Let $Z=\pi^{-1}(\omega)$, where $\pi$ is the projection from $X$ to $\omega+1$. Then $Z \in Z(X)$ and $x_{\infty} \in \mathrm{cl}_{\beta X} Z$. Define a map $\psi: Z \rightarrow D_{0}$ by $\psi((b, \omega, \xi))=\xi$. Then $\psi$ is a closed, continuous map, and for each $\xi \in D_{0}, x_{\infty} \notin \mathrm{cl}_{\beta X} f^{-1}(\xi)$, since $G(0,0, \xi) \cap f^{-1}(\xi)=\varnothing$. Clearly $D_{0}$ is a paracompact $D$-space. Hence it follows from Claim 6 and Corollary 3.2 that $X$ is not a quasi-Maŕík space. This completes the whole proof.

We turn to answer question E. By [28, Proposition 4.4], the absolute $E(X)$ of a countably paracompact space $X$ is cozero-dominated and hence a Mařík space. Therefore, Example 3.5 also provides a negative answer to question $\mathrm{E}$. Another counterexample is the Dieudonne Plank $D$. In his earlier paper [27], Wheeler proved that $E(D)$ is $\mathrm{MC}$, while $D$ is not a Marík space. However, neither example is locally compact. Here we show that the Dieudonné Plank can easily be modified to a locally compact space.

Example 3.6. There exists a locally compact space $Y$ which is not a quasi-Maríik space, such that $E(Y)$ is cozero-dominated and MC.

Proof. Let $\alpha D=D \cup\{\infty\}$ be the one-point compactification of a discrete space $D$ of cardinality $\omega_{1}$. Define

$$
Y=(\alpha D \times(\omega+1))-\{(\infty, \omega)\} .
$$

Clearly $Y$ is locally compact. Let $Z=D \times\{\omega\}$. For each $B \in \mathrm{Ba}(Y)$, it can easily be checked that either $|B \cap Z| \leq \omega$ or $|Z-B| \leq \omega$. Define a Baire measure $\mu$ on $Y$ by $\mu(B)=0$ in the former case and $\mu(B)=1$ in the latter case. Then there exists $y \in v Y-Y$ such that $S\left(\mu^{\beta}\right)=\{y\}$. Since $Z$ is a discrete zero-set with $y \in \mathrm{cl}_{\beta X} Z$, it follows from Corollary 3.3 that $Y$ is not a quasi-Maŕik space. To see that $E(Y)$ is cozero-dominated and MC, let $X$ be the space defined in the proof of Example 2.4. Then the natural map $\phi: X \rightarrow Y$ collapsing the set $(\beta D-D) \times\{n\}$ to the point $(\infty, n)$ for each $n \in \omega$ is perfect irreducible. Thus, by the uniqueness of the absolute, $E(X)=E(Y)$. As we have 
proved in 2.4 and $2.5, X$ is cozero-dominated and MC, and hence so is $E(Y)$ by [27, Theorem 2] and [28, Remark 4.3].

The space $\Psi=N \cup \mathscr{R}$, described in [11, 5I, p. 79], is a locally compact, pseudocompact space in which $N$ is dense, and $\mathscr{R}$ is a discrete zero-set with $|\mathscr{R}| \leq \mathfrak{c}$. Since all subsets of $\Psi$ are Borel sets, $\Psi$ is weakly Borel measure complete if $c<m_{r}$. In [19], Mrówka proved that $\mathscr{R}$ can be chosen so that $|\beta \Psi-\Psi|=1$. We use this $\Psi$ to show that $E(Y)$ need not be a Maŕík space even if $Y$ is.

Example 3.7. Assume $\mathfrak{c}<m_{r}$. Then there exists a pseudocompact, locally compact, Mařík space $Y$ for which $E(Y)$ is not a quasi-Mařík space.

Proof. Let $\Psi$ be the space due to Mrówka stated above, and let $\beta \Psi-\Psi=\{p\}$. Define

$$
\begin{aligned}
& S=(\beta \Psi \times(\omega+1))-\{(p, \omega)\}, \\
& T=\left(\left(\omega_{1}+1\right) \times(\omega+1)\right)-\left\{\left(\omega_{1}, \omega\right)\right\},
\end{aligned}
$$

and $X=S \oplus T$, where $\oplus$ means the topological sum. The desired space $Y$ is the quotient space obtained from $X$ by identifying points $(p, n)$ with $\left(\omega_{1}, n\right)$ for each $n \in \omega$. Let $\phi: X \rightarrow Y$ be the quotient map. Since $\Psi$ is pseudocompact and locally compact, so is $Y$. First, we show that $Y$ is a Maŕik space. Although the proof is essentially the same as the proof, due to Wheeler [28, p. 101], that his space $T \# D$ is a Maŕík space, we do this in detail for the convenience of the reader. Observe that $|\beta Y-Y|=1$, and let $\beta Y-Y=\{y\}$. Since $Y$ is pseudocompact, $\beta Y=v Y$. Let $\mu$ be a Baire measure on $Y$. We have to prove that $\mu$ extends to a regular Borel measure. Let

$$
r=\inf \left\{\mu(U): y \in U^{\beta}, U \in \operatorname{Coz}(Y)\right\},
$$

where $U^{\beta}$ is the unique Baire set of $\beta Y(=v Y)$ with $U=U^{\beta} \cap Y$. For each $B \in \mathrm{Ba}(Y)$, define $\mu_{1}(B)=r$ if $y \in B^{\beta}$, and $\mu_{1}(B)=0$ otherwise. Then $\mu_{1}$ is a Baire measure on $Y$. Define $\mu_{2}=\mu-\mu_{1}$. Then, since $\inf \left\{\mu_{2}(U): y \in\right.$ $\left.U^{\beta}, U \in \operatorname{Coz}(Y)\right\}=0, \mu_{2}$ is $\tau$-additive by [15, Theorem 2.4], and hence $\mu_{2}$ has a regular Borel extension $\nu_{2}$ (see $[15$, p. 144]). On the other hand, let

$$
\mathscr{F}=\left\{\phi(E \times\{\omega\}): E \text { is a closed unbounded set of } \omega_{1}\right\} .
$$

For each $A \in \operatorname{Bo}(Y)$, either $A$ or $Y-A$ contains a set $F \in \mathscr{F}$. Define $\nu_{1}(A)=r$ if $A$ contains a set $F \in \mathscr{F}$, and $\nu_{1}(A)=0$ otherwise. Then $\nu_{1}$ is a regular Borel extension of $\mu_{1}$. Consequently, $\mu$ extends to a regular Borel measure $\nu=\nu_{1}+\nu_{2}$ on $Y$. Hence $Y$ is proved to be a Marrík space. Next, we show that $E(Y)$ is not a quasi-Mařik space. Since $\phi$ is perfect irreducible, $E(X)=E(Y)$, and $E(X)=E(S) \oplus E(T)$. It is known [22, Theorem 3.3 and 3, Corollary 4.13] that weakly Borel measure complete spaces are preserved by countable unions and perfect preimages. Using these results, we can check that $E(S)$ is weakly Borel measure complete. Since $X$ is pseudocompact, so is $E(X)$ by [30, Proposition 2.5], and hence $\beta E(X)=v E(X)$. Consequently,

$$
\mathrm{cl}_{\beta E(X)} E(S) \cap(v E(X)-E(X)) \neq \varnothing .
$$


Hence it follows from Corollary 3.3 and Remark 3.4 that $E(X)$, and hence $E(Y)$, is not a quasi-Maŕík space.

Remark 3.8. That the space $\Psi$ is not a quasi-Maŕík space under $c<m_{r}$ was first observed by Adamski in [1]. This provides a negative answer to another Wheeler's question [29, Problem 9.16], whether every pseudocompact space is a Maŕik space. The following question is yet unanswered.

Question 3.9. Is there a pseudocompact space which is not a (quasi-) Marrik space without assuming $\mathfrak{c}<m_{r}$ ?

\section{TOPOlOGICAL PROPERTIES OF MǍ̌́fK SPACES}

Generally speaking, Mařík spaces are badly behaved under topological operations. We can, however, prove a few positive results. We begin by considering how Mařik spaces are preserved in subspaces. A subset $S \subset X$ is called a generalized Baire set if for each open set $G$ with $S \subset G$, there exists $B \in \mathrm{Ba}(X)$ such that $S \subset B \subset G$.

Theorem 4.1. Let $X$ be a Mařik space and $Y$ a Baire-embedded, generalized Baire set of $X$. Then $Y$ is a Mařik space.

Proof. Let $\mu$ be a Baire measure on $Y$. For each $A \in \mathrm{Ba}(X)$, define $\mu_{X}(A)=$ $\mu(A \cap Y)$. Then $\mu_{X}$ is a Baire measure on $X$, and hence $\mu_{X}$ extends to a regular Borel measure $\nu_{X}$ on $X$. For each $B \in \operatorname{Bo}(Y)$, define

$$
\nu(B)=\inf \left\{\nu_{X}(G): B \subset G, G \text { is open in } X\right\} .
$$

Then, by [10, Proposition 3.6], $\nu$ is a regular Borel measure on $Y$. Since $Y$ is a Baire-embedded, generalized Baire set, it follows from the next lemma that $\nu$ is an extension of $\mu$.

Lemma 4.2. Let $\mu$ and $\nu$ be the same as above, and let $Y$ be the generalized Baire set. Then, for each $A \in \mathrm{Ba}(X), \mu(A \cap Y)=\nu(A \cap Y)$.

Proof. Let $A \in \mathrm{Ba}(X)$. Then

$$
\begin{aligned}
\mu(A \cap Y) & =\mu_{X}(A)=\inf \left\{\mu_{X}(U): A \subset U \in \operatorname{Coz}(X)\right\} \\
& =\inf \left\{\nu_{X}(U): A \subset U \in \operatorname{Coz}(X)\right\} \geq \nu(A \cap Y) .
\end{aligned}
$$

To prove the converse, let $\varepsilon>0$. Then there exists $Z \in Z(X)$ such that $Z \subset A$ and $\mu_{X}(A)-\varepsilon<\mu_{X}(Z)$. For each open set $G$ in $X$ with $A \cap Y \subset G$, by the condition of $Y$ there exists $J \in \mathrm{Ba}(X)$ such that $Y \subset J$ and $J \cap(Z-G)=\varnothing$. Define $Z_{0}=Z \cap J$; then $Z_{0} \in \mathrm{Ba}(X)$ and $Z_{0} \subset G$. Since $Z_{0} \cap Y=Z \cap Y$,

$$
\mu_{X}(Z)=\mu_{X}\left(Z_{0}\right)=\nu_{X}\left(Z_{0}\right) \leq \nu_{X}(G),
$$

so $\mu(A \cap Y)-\varepsilon=\mu_{X}(A)-\varepsilon<\nu_{X}(G)$. Since $G$ and $\varepsilon$ are arbitrary, it follows that $\mu(A \cap Y) \leq \nu(A \cap Y)$.

Corollary 4.3. Let $X$ be a Mař́k space and $Y$ a cozero-set of $X$. Then $Y$ is a Mařik space. 
Remarks 4.4. (1) As Wheeler mentioned in [29], the space $T \# D$ in [28, p. 101] shows that a $C$-embedded, regular closed subspace of a Maŕík space need not be a Mařík space (see also Example 3.7).

(2) We do not know whether the assumption in Theorem 4.1 that $Y$ is Baireembedded can be removed.

Recall from [2] that a space $X$ is Baire-separated if for each pair $F_{1}, F_{2}$ of disjoint closed sets, there exists $B \in \mathrm{Ba}(X)$ such that $F_{1} \subset B$ and $B \cap F_{2}=\varnothing$. All normal spaces are Baire-separated, but the converse is not true. For example, the space $X$ defined in the proof of Example 2.3 is certainly the case. The proof of the following lemma is left to the reader since it is routine.

Lemma 4.5. For a space $X$, the following conditions are equivalent:

(a) $X$ is Baire-separated.

(b) For each pair $F_{1}, F_{2}$ of disjoint $F_{\sigma}$-sets of $X$, there exists $B \in \mathrm{Ba}(X)$ such that $F_{1} \subset B$ and $B \cap F_{2}=\varnothing$.

(c) Every $F_{\sigma}$-set of $X$ is a generalized Baire set.

(d) Every $F_{\sigma}$-set of $X$ is Baire-embedded in $X$.

Theorem 4.6. Let $X$ be a Baire-separated, Mařik space and $Y$ a generalized Baire set of $X$. Then $Y$ is a Mařik space.

Proof. Let $\mu$ be a Baire measure on $Y$. Let $\mu_{X}, \nu_{X}$, and $\nu$ be the same as in the proof of Theorem 4.1. We have to prove that $\nu$ is an extension of $\mu$. To do this, let $B \in \mathrm{Ba}(Y)$. For each $i \in N$, by the regularity of $\nu$, there exist a closed set $F_{i}$ and an open set $G_{i}$ in $Y$ such that $F_{i} \subset B \subset G_{i}$ and

$$
\nu(B)-1 / i<\nu\left(F_{i}\right) \leq \nu\left(G_{i}\right)<\nu(B)+1 / i .
$$

Similarly, we can choose $Z_{i} \in Z(Y)$ and $U_{i} \in \operatorname{Coz}(Y)$ such that $Z_{i} \subset B \subset U_{i}$ and

$$
\mu(B)-1 / i<\mu\left(Z_{i}\right) \leq \mu\left(U_{i}\right)<\mu(B)+1 / i .
$$

We may assume that $\left\{F_{i}\right\}$ and $\left\{Z_{i}\right\}$ are increasing and $\left\{G_{i}\right\}$ and $\left\{U_{i}\right\}$ are decreasing. For each $i \in N$, there exist a closed set $E_{i}$ in $X$ with $E_{i} \cap Y=$ $F_{i} \cup Z_{i}$ and an open set $H_{i}$ in $X$ with $H_{i} \cap Y=G_{i} \cap U_{i}$. Since $Y$ is a generalized Baire set, there exists $J_{i} \in \mathrm{Ba}(X)$ such that $Y \subset J_{i}$ and $J_{i} \cap\left(E_{i}-H_{i}\right)=\varnothing$. Let us set $J=\bigcap_{i \in N} J_{i}$. Then $Y \subset J \in \mathrm{Ba}(X)$, so $\mu_{X}(J)=\mu(Y)$. For each $i \in N$, take $K_{i} \in Z(X)$ such that $K_{i} \subset J$ and $\mu_{X}(J)-1 / i<\mu_{X}\left(K_{i}\right)$, and define $K=\bigcup_{i \in N} K_{i}$. Then, since $\mu_{X}(J-K)=0, \mu(Y-K)=0$. On the other hand, since

$$
\nu(Y-K) \leq \nu_{X}\left(X-K_{i}\right)=\mu_{X}\left(X-K_{i}\right)<1 / i
$$

for each $i \in N, \nu(Y-K)=0$. Thus

$$
\nu(Y-K)=\mu(Y-K)=0 .
$$

Since $E_{i} \cap K \subset H_{i} \cap K$, by Lemma 4.5 there exists $A_{i} \in \mathrm{Ba}(X)$ such that $E_{i} \cap K \subset A_{i}$ and $A_{i} \cap\left(K-H_{i}\right)=\varnothing$. Then, for each $i \in N$,

$$
\left(F_{i} \cup Z_{i}\right) \cap K \subset A_{i} \cap K \cap Y \subset\left(G_{i} \cap U_{i}\right) \cap K .
$$


Define $A_{*}=\bigcup_{j \in N}\left(\bigcap_{i \geq j}\left(A_{i} \cap K \cap Y\right)\right)$ and $A^{*}=\bigcap_{j \in N}\left(\bigcup_{i \geq j}\left(A_{i} \cap K \cap Y\right)\right)$; then

$$
\left(\bigcup_{i \in N} F_{i}\right) \cap K \subset A_{*} \subset A^{*} \subset\left(\bigcap_{i \in N} G_{i}\right) \cap K .
$$

Since $\nu\left(\left(\bigcup_{i \in N} F_{i}\right) \cap K\right)=\nu(B)=\nu\left(\left(\cap_{i \in N} G_{i}\right) \cap K\right)$ by (1) and (2),

$$
\begin{aligned}
\nu(B) & \leq \nu\left(A_{*}\right) \leq \liminf \nu\left(A_{i} \cap K \cap Y\right) \\
& \leq \lim \sup \nu\left(A_{i} \cap K \cap Y\right) \leq \nu\left(A^{*}\right) \leq \nu(B),
\end{aligned}
$$

and hence

$$
\nu(B)=\lim _{i \rightarrow \infty} \nu\left(A_{i} \cap K \cap Y\right) .
$$

Similarly,

$$
\mu(B)=\lim _{i \rightarrow \infty} \mu\left(A_{i} \cap K \cap Y\right) .
$$

Since $A_{i} \cap K \in \mathrm{Ba}(X)$, it follows from Lemma 4.2 that $\nu\left(A_{i} \cap K \cap Y\right)=$ $\mu\left(A_{i} \cap K \cap Y\right)$ for each $i \in N$. Consequently, $\nu(B)=\mu(B)$, which completes the proof.

Corollary 4.7. Let $X$ be a Baire-separated, Mařik space, and let $Y$ be either an $F_{\sigma}$-set or a Baire set of $X$. Then $Y$ is a Marík space.

Remarks 4.8. (1) In Theorem 4.6, "generalized Baire" cannot be replaced by "open." Consider a locally compact, non-Maŕik space $X$ (see Example 3.6) as a subspace of $\beta X$.

(2) A Baire set of a Baire-separated, Mařík space need not be Baire-embedded, so Theorem 4.6 cannot be reduced to Theorem 4.1. To see this, let $M$ be the Michael line defined in $\S 2$ and $P$ the subspace of irrational numbers. Being paracompact, $M$ is a Baire-separated, Mařík space, and $P \in \mathrm{Ba}(M)$. We show that $P$ is not Baire-embedded in $M$. Let $\left\{P_{1}, P_{2}\right\}$ be a partition of $P$ with the usual topology such that $U \cap P_{i} \in \mathscr{C}_{2}(P)$ for each nonempty open set $U \subset P$ and $i=1,2$. The existence of such a partition can be shown similarly to Claim 1 in the proof of Theorem 2.5. Since $P$ is discrete in $M, P_{1}$ is a Baire set of $P$ in $M$. Suppose that there exists $A \in \mathrm{Ba}(M)$ with $P_{1}=A \cap P$. Then, since $P_{1} \subset A$, it follows from Corollary 2.9 that $P-A \in \mathscr{C}_{1}(P)$. But $P-A=P_{2} \in \mathscr{C}_{2}(P)$, a contradiction. This also shows that, in Lemma 4.5, " $F_{\sigma}$-set" cannot be replaced by "Baire-set."

We now turn to the preservation under taking unions.

Theorem 4.9. Assume that $X=\bigcup_{n \in N} X_{n}$, and each $X_{n}$ is a Mařik space and is a Baire-embedded, generalized Baire set of $X$. Then $X$ is a Mařik space.

Proof. Let $\mu$ be a Baire measure on $X$. For each $n \in N$ and each $B \in \operatorname{Ba}\left(X_{n}\right)$, define

$$
\mu_{n}(B)=\inf \{\mu(U): B \subset U \in \operatorname{Coz}(X)\} .
$$

Since $X_{n}$ is Baire-embedded, $\mu_{n}$ is a Baire-measure on $X_{n}$, and hence $\mu_{n}$ extends to a regular Borel measure $\xi_{n}$ on $X_{n}$. For each $i \in N$, take $J_{n i} \in$ $\operatorname{Coz}(X)$ such that $X_{n} \subset J_{n i}$ and $\mu\left(J_{n i}\right)<\mu_{n}\left(X_{n}\right)+1 / i$, and define $J_{n}=$ 
$\bigcap_{i \in N} J_{n i}$. Then $X_{n} \subset J_{n} \in \operatorname{Ba}(X)$ and $\mu\left(J_{n}\right)=\mu_{n}\left(X_{n}\right)$. For each $C \in \operatorname{Bo}\left(J_{n}\right)$, define $\nu_{n}(C)=\xi_{n}\left(C \cap X_{n}\right)$. Then $\nu_{n}$ is a Borel measure on $J_{n}$.

Claim 1. For each $A \in \mathrm{Ba}(X), \nu_{n}\left(A \cap J_{n}\right)=\mu\left(A \cap J_{n}\right)$.

Proof. It is easily checked that $\nu_{n}\left(A \cap J_{n}\right) \leq \mu\left(A \cap J_{n}\right)$. To prove the converse, let $\varepsilon>0$. Then there exists $V \in \operatorname{Coz}(X)$ such that $A \cap X_{n} \subset V$ and $\mu(V)<$ $\mu_{n}\left(A \cap X_{n}\right)+\varepsilon=\nu_{n}\left(A \cap J_{n}\right)+\varepsilon$. Let $W=\left(A \cap J_{n}\right)-V$. Then $\mu(W)=0$ by the definition of $J_{n}$. Since $A \cap J_{n} \subset V \cup W, \mu\left(A \cap J_{n}\right) \leq \mu(V \cup W)=\mu(V)$, and hence $\mu\left(A \cap J_{n}\right) \leq \nu_{n}\left(A \cap J_{n}\right)$.

Claim 2. For each $D \in \operatorname{Bo}\left(J_{n}\right), \nu_{n}(D)=\sup \left\{\nu_{n}(F): F \subset D\right.$ and $F$ is closed in $X$ \} .

Proof. We first prove that $\nu_{n}$ is a regular measure on $J_{n}$. For this end, by [10, Proposition 6.2], it suffices to prove that for each open set $G$ in $J_{n}$,

$$
\nu_{n}(G)=\sup \left\{\nu_{n}(H): H \subset G \text { and } H \text { is closed in } J_{n}\right\} .
$$

Let $\varepsilon>0$. Since $\xi_{n}$ is regular, there exists a closed set $H^{\prime}$ in $X_{n}$ such that $H^{\prime} \subset G \cap X_{n}$ and

$$
\xi_{n}\left(H^{\prime}\right)>\xi_{n}\left(G \cap X_{n}\right)-\varepsilon / 2=\nu_{n}(G)-\varepsilon / 2 .
$$

Since $X_{n}$ is a generalized Baire set, there exists $K \in \mathrm{Ba}(X)$ such that $X_{n} \subset K$ and $K \cap\left(\mathrm{cl}_{X} H^{\prime}-G_{0}\right)=\varnothing$, where $G_{0}$ is an open set in $X$ with $G_{0} \cap J_{n}=G$. Take $Z \in Z(X)$ such that $Z \subset K$ and $\mu(K-Z)<\varepsilon / 2$, and define $H=$ $\mathrm{cl}_{X} H^{\prime} \cap Z \cap J_{n}$. Then $H$ is closed in $J_{n}$ and $H \subset G$. Since $H^{\prime} \cap Z=H \cap X_{n}$ and $H^{\prime}-Z \subset K-Z$,

$$
\begin{aligned}
\xi_{n}\left(H^{\prime}\right) & =\xi_{n}\left(H^{\prime} \cap Z\right)+\xi_{n}\left(H^{\prime}-Z\right) \\
& \leq \xi_{n}\left(H \cap X_{n}\right)+\mu(K-Z)<\nu_{n}(H)+\varepsilon / 2 .
\end{aligned}
$$

It follows from (1) and (2) that $\nu_{n}(H)>\nu_{n}(G)-\varepsilon$. Thus $\nu_{n}$ is proved to be regular. Let $D \in \operatorname{Bo}\left(J_{n}\right)$, and let $\varepsilon>0$ again. Since $\nu_{n}$ is regular, there exists a closed set $F^{\prime}$ in $J_{n}$ such that $F^{\prime} \subset D$ and $\nu_{n}\left(F^{\prime}\right)>\nu_{n}(D)-\varepsilon / 2$. Take $Z^{\prime} \in Z(X)$ such that $Z^{\prime} \subset J_{n}$ and $\mu\left(J_{n}-Z^{\prime}\right)<\varepsilon / 2$, and define $F=F^{\prime} \cap Z^{\prime}$. Then $F$ is closed in $X, F \subset D$, and by Claim 1

$$
\begin{aligned}
\nu_{n}(F) & \geq \nu_{n}\left(F^{\prime}\right)-\nu_{n}\left(J_{n}-Z^{\prime}\right) \\
& >\left(\nu_{n}(D)-\varepsilon / 2\right)-\mu\left(J_{n}-Z^{\prime}\right)>\nu_{n}(D)-\varepsilon,
\end{aligned}
$$

thus proving the claim.

To complete the proof, let $Y_{n}=J_{n}-\bigcup_{i<n} J_{i}$ for each $i \in N$. Then $X=$ $\bigcup_{n \in N} Y_{n}, Y_{n} \in \mathrm{Ba}(X)$, and $Y_{n} \cap Y_{m}=\varnothing$ if $n \neq m$. For each $E \in \operatorname{Bo}(X)$, define $\nu(E)=\sum_{n \in N} \nu_{n}\left(E \cap Y_{n}\right)$. Then it follows from Claims 1 and 2 that $\nu$ is a regular Borel extension of $\mu$.

Corollary 4.10. Assume that $\mathscr{U}$ is a locally finite cover of a space $X$ by cozerosets such that each $U \in \mathscr{U}$ is a Mařik space and $|\mathscr{U}|<m_{r}$. Then $X$ is a Mařik space. 
Proof. By [21, Theorem 1.2], $\mathscr{U}$ has a refinement $\mathscr{V}=\bigcup_{n \in N} \mathscr{V}_{n}$ by cozerosets such that each $\mathscr{V}_{n}=\left\{V_{\lambda}: \lambda \in \Lambda_{n}\right\}$ is discrete. For each $n \in N$, let $X_{n}=\bigcup_{\lambda \in \Lambda_{n}} V_{\lambda}$; then $X=\bigcup_{n \in N} X_{n}$ and $X_{n} \in \operatorname{Coz}(X)$. By Theorem 4.9, it suffices to prove that each $X_{n}$ is a Maŕik space. For this end, let $\mu$ be a Baire measure on $X_{n}$. Since $\mu$ is finite, there exists a countable set $M \subset \Lambda_{n}$ such that $\mu\left(V_{\lambda}\right)=0$ if $\lambda \in \Lambda_{n}-M$. Define $Y_{n}=\bigcup_{\lambda \in M} V_{\lambda}$. Then $Y_{n} \in \operatorname{Coz}(X)$ and $Y_{n}$ is a Maŕík space by Theorem 4.9, so $\mu \mid \mathrm{Ba}\left(Y_{n}\right)$ extends to a regular Borel measure $\nu_{n}$ on $Y_{n}$. Since $\left|\Lambda_{n}-M\right|<m_{r}, \mu\left(X_{n}-Y_{n}\right)=0$. Consequently, if we define $\nu(B)=\nu_{n}\left(B \cap Y_{n}\right)$ for each $B \in \operatorname{Bo}\left(X_{n}\right)$, then $\nu$ is a regular Borel extension of $\mu$.

Corollary 4.11. Assume that $X=\bigcup_{n \in N} X_{n}$ is a Baire-separated space, and each $X_{n}$ is a Mařik space and is either a closed set or a Baire set of $X$. Then $X$ is a Mařik space.

Proof. By the proof of Theorem 4.9, it suffices to prove that for each $n \in N$, there exist $J_{n} \in \mathrm{Ba}(X)$ with $X_{n} \subset J_{n}$ and a Borel measure $\nu_{n}$ on $J_{n}$ satisfying the Claims 1 and 2. In case $X_{n}$ is closed, we can define such $J_{n}$ and $\nu_{n}$ quite similarly since $X_{n}$ is then a Baire-embedded, generalized Baire set by Lemma 4.5. In case $X_{n} \in \mathrm{Ba}(X)$, define $J_{n}=X_{n}$. For each $i \in N$, take $K_{i} \in Z(X)$ such that $K_{i} \subset J_{n}$ and $\mu\left(K_{i}\right)>\mu\left(J_{n}\right)-1 / i$, and define $K=\bigcup_{i \in N} K_{i}$. Since $K$ is Baire-embedded by Lemma 4.5, it follows from Theorem 4.1 that $K$ is a Maŕik space. Hence, if we consider $K$ instead of $X_{n}$, then we can define $\nu_{n}$ on $J_{n}$ similarly to the proof of Theorem 4.9.

Remarks 4.12. (1) The space $Y$ defined in the proof of Example 3.6 shows that the union of two Mařík spaces need not be a quasi-Mařik space even if one is a cozero-set and the other is a zero-set.

(2) We do not know whether the assumption in Theorem 4.9 that each $X_{n}$ is a generalized Baire set can be removed. The assumption was used only to ensure the regularity of the extension $\nu$. Thus $X$ is a quasi-Marík space even if each $X_{n}$ is only assumed to be Baire-embedded.

Question 4.13. Let $X=Y \cup K$ be the union of a Mařik space $Y$ with a compact space $K$. Then is $X$ is Maŕik space?

Question 4.14. Let $X=\bigoplus_{\lambda \in \Lambda} X_{\lambda}$ be the disjoint sum of Mařík spaces $X_{\lambda}$, $\lambda \in \Lambda$. Then is $X$ a Marík space even if $|\Lambda|$ is real-valued measurable?

Finally we are concerned with the preservation under maps and products. Examples 3.6 and 3.7 show that the image and the preimage of Maŕik spaces under perfect maps need not be quasi-Maŕik spaces, respectively. If we make some additional assumptions, then Mařík spaces are preserved under perfect maps. To show this, we need a theorem due to Bachman and Sultan [4]. Before stating their theorem, let us agree on some terminology. Let $\mathscr{L}_{1} \subset \mathscr{L}_{2}$ be two lattices, closed under countable intersections, of subsets of a set $X$, and let $\mathscr{A}\left(\mathscr{L}_{i}\right)$ denote the smallest algebra containing $\mathscr{L}_{i}, i=0,1$. Then $\mathscr{L}_{2}$ is said to be $\mathscr{A}\left(\mathscr{L}_{1}\right)$-countably paracompact if $A_{n} \downarrow \varnothing$ in $\mathscr{L}_{2}$ implies the existence of 
a sequence $\left\{B_{n}\right\}_{n \in \omega} \subset \mathscr{A}\left(\mathscr{L}_{1}\right)$ such that $A_{n} \subset B_{n}$ and $B_{n} \downarrow \varnothing$. A (finitely additive) measure $\mu$ defined on $\mathscr{A}\left(\mathscr{L}_{i}\right)$ is called $\mathscr{L}_{i}$-regular if for each $B \in$ $\mathscr{A}\left(\mathscr{L}_{i}\right), \mu(B)=\sup \left\{\mu(A): A \subset B, A \in \mathscr{L}_{i}\right\}$.

Bachman-Sultan's extension theorem. Let $\mathscr{L}_{1} \subset \mathscr{L}_{2}$ be the same as above. Then every finitely additive, $\mathscr{L}_{1}$-regular measure $\mu$ defined on $\mathscr{A}\left(\mathscr{L}_{1}\right)$ can be extended to a finitely additive, $\mathscr{L}_{2}$-regular measure $\nu$ defined on $\mathscr{A}\left(\mathscr{L}_{2}\right)$. If $\mathscr{L}_{2}$ is $\mathscr{A}\left(\mathscr{L}_{1}\right)$-countably paracompact and if $\mu$ is $\sigma$-additive, then so is $\nu$.

For a space $X, \mathrm{Ba}^{\omega}(X)\left(\mathrm{Bo}^{\omega}(X)\right)$ denotes the smallest algebra containing all zero-sets (closed sets) of $X$. It is well known (cf. [13, 10.36]) that every measure $\mu$ defined on $\mathrm{Ba}^{\omega}(X) \quad\left(\mathrm{Bo}^{\omega}(X)\right)$ can be extended to a unique Baire (Borel) measure $\nu$ on $X$, and if $\mu$ is regular, then so is $\nu$.

Theorem 4.15. Let $f$ be a continuous map from a Mařik space $X$ onto a space $Y$ such that for each $Z \in Z(X), f(Z) \in Z(Y)$, and such that for each $y \in Y$, $f^{-1}(y)$ is relatively pseudocompact in $X$; i.e., each $g \in C(X)$ is bounded on $f^{-1}(y)$. Then $Y$ is a Mařik space.

Proof. Let $\mu$ be a Baire measure on $Y$. Define $\mathscr{L}_{1}=\left\{f^{-1}(Z): Z \in Z(Y)\right\}$ and $\mathscr{L}_{2}=Z(X)$. Then $\mathscr{L}_{1} \subset \mathscr{L}_{2}$ and $\mathscr{A}\left(\mathscr{L}_{1}\right)=\left\{f^{-1}(B): B \in \mathrm{Ba}^{\omega}(Y)\right\}$. For each $B \in \mathrm{Ba}^{\omega}(Y)$, define $\lambda\left(f^{-1}(B)\right)=\mu(B)$. Then $\lambda$ is a $\mathscr{L}_{1}$-regular measure defined on $\mathscr{A}\left(\mathscr{L}_{1}\right)$. By the condition of $f$, it is easily checked that $\mathscr{L}_{2}$ is $\mathscr{A}\left(\mathscr{L}_{1}\right)$-countably paracompact, and hence it follows from BachmanSultan's theorem that $\lambda$ can be extended to an $\mathscr{L}_{2}$-regular measure $\lambda_{1}$ defined on $\mathscr{A}\left(\mathscr{L}_{2}\right) \quad\left(=\mathrm{Ba}^{\omega}(X)\right)$. Since $\lambda_{1}$ extends to a Baire measure on $X$ and $X$ is a Maŕik space, $\lambda$ extends to a regular Borel measure $\xi$ on $X$. For each $A \in \operatorname{Bo}(Y)$, define $\nu(A)=\xi\left(f^{-1}(A)\right)$. Then, since $\mu\left|\mathrm{Ba}^{\omega}(Y)=\nu\right| \mathrm{Ba}^{\omega}(Y)$, $\mu=\nu \mid \mathrm{Ba}(Y)$, and hence $\nu$ is a regular Borel extension of $\mu$.

Corollary 4.16. Let $f$ be an open, perfect map from a Mařik space $X$ onto a space $Y$. Then $Y$ is a Mař̀k space.

Proof. By [8, Lemma 3.4], an open perfect map carries a zero-set to a zero-set. Hence this follows from Theorem 4.15.

Theorem 4.17. Let $f$ be a closed, continuous map from a space $X$ onto a Baireseparated, Mařik space $Y$ such that for each $y \in Y, f^{-1}(y)$ is countably compact. Then $X$ is a Mařik space.

Proof. Let $\mu$ be a Baire measure on $X$. We have to prove that $\mu$ admits a regular Borel extension. For each $B \in \mathrm{Ba}(Y)$, define $\lambda(B)=\mu\left(f^{-1}(B)\right)$; then $\lambda$ is a Baire measure on $Y$. Since $Y$ is a Mařik space, $\lambda$ extends to a regular Borel measure $\xi$ on $Y$. On the other hand, by Bachman-Sultan's theorem, $\mu \mid \mathrm{Ba}^{\omega}(X)$ extends to a finitely additive, regular measure $\nu_{1}$ defined on $\mathrm{Bo}^{\omega}(X)$. For each $A \in \mathrm{Bo}^{\omega}(Y)$, define $\xi_{1}(A)=\nu_{1}\left(f^{-1}(A)\right)$. Then $\xi_{1}$ is regular. We now prove that

$$
\xi_{1}=\xi \mid \mathrm{Bo}^{\omega}(Y)
$$


Since both $\xi$ and $\xi_{1}$ are regular, it suffices to show that they coincide on open sets. To do this, let $G \subset Y$ be open and let $\varepsilon>0$. Then there exists a closed set $E \subset G$ such that $\xi(E)>\xi(G)-\varepsilon$. Since $Y$ is Baire-separated, there exists $J \in \mathrm{Ba}(Y)$ with $E \subset J \subset G$. Then

$$
\begin{aligned}
\xi(J) & =\lambda(J)=\sup \{\lambda(Z): Z \subset J, Z \in Z(Y)\} \\
& =\sup \left\{\xi_{1}(Z): Z \subset J, Z \in Z(Y)\right\} \leq \xi_{1}(G),
\end{aligned}
$$

so $\xi(G)-\varepsilon<\xi(J) \leq \xi_{1}(G)$, and hence $\xi(G) \leq \xi_{1}(G)$. Conversely, let $\varepsilon>0$ again, and take a closed set $F \subset G$ and $K \in \mathrm{Ba}(Y)$ such that $\xi_{1}(F)>\xi_{1}(G)-\varepsilon$ and $F \subset K \subset G$. Then

$$
\begin{aligned}
\xi_{1}(F) & =\nu_{1}\left(f^{-1}(F)\right) \leq \inf \left\{\nu_{1}(V): f^{-1}(K) \subset V \in \operatorname{Coz}(X)\right\} \\
& =\mu\left(f^{-1}(K)\right)=\lambda(K)=\xi(K),
\end{aligned}
$$

so $\xi_{1}(G)-\varepsilon<\xi(K) \leq \xi(G)$, and hence $\xi_{1}(G) \leq \xi(G)$. Thus (1) is proved. To see that $\nu_{1}$ is $\sigma$-additive, let $\left\{F_{n}\right\}_{n \in \omega}$ be a sequence of closed sets in $X$ with $F_{n} \downarrow \varnothing$. By the condition of $f, f\left(F_{n}\right)$ is closed and $f\left(F_{n}\right) \downarrow \varnothing$. Since $\xi$ is $\sigma$-additive, it follows from (1) that

$$
\lim _{n \rightarrow \infty} \nu_{1}\left(F_{n}\right) \leq \lim _{n \rightarrow \infty} \nu_{1}\left(f^{-1}\left(f\left(F_{n}\right)\right)\right)=\lim _{n \rightarrow \infty} \xi\left(f\left(F_{n}\right)\right)=0 .
$$

Consequently, $\nu_{1}$ is $\sigma$-additive, and hence $\nu_{1}$ can be extended to a regular Borel measure on $X$, which is a required extension of $\mu$.

Corollary 4.18. Let $Y$ be a Baire-separated, Mařik space. Then the absolute $E(Y)$ is a Mařik space.

Corollary 4.19. Let $X$ be a Baire-separated, Mařik space and $Y$ a compact space. Then $X \times Y$ is a Mařik space.

Example 3.7 shows that the assumptions in Theorem 4.17 and Corollary 4.18 that $Y$ is Baire-separated cannot be removed; however, the following questions remain unanswered.

Questions 4.20. Is the product of a Mařík space with a compact space a Mařík space? More generally, is the preimage of a Mařík space under an open, perfect map a Mařik space?

\section{REFERENCES}

1. W. Adamski, $\tau$-smooth Borel measures on topological spaces, Math. Nachr. 78 (1977), 97-107.

2. Extensions of tight set functions with applications in topological measure theory, Trans. Amer. Math. Soc. 283 (1984), 353-368.

3. G. Bachman and A. Sultan, Measure theoretic techniques in topology and mappings of replete and measure replete spaces, Bull. Austral. Math. Soc. 18 (1978), 267-285.

4. __, On regular extensions of measures, Pacific J. Math. 86 (1980), 389-395.

5. W. W. Comfort and S. Negrepontis, Continuous pseudometrics, Marcel Dekker, New York, 1975.

6. R. Engelking, General topology, Polish Scientific Publishers, Warszawa, 1977. 
7. D. H. Fremlin, Uncountable powers of $R$ can be almost Lindelöf, Manuscripta Math. 22 (1977), 77-85.

8. Z. Frolik, Applications of complete families of continuous functions to the theory of $Q$-spaces, Czechoslovak Math. J. 11 (1961), 115-133.

9. R. J. Gardner, The regularity of Borel measures and Borel measure-compactness, Proc. London Math. Soc. 30 (1975), 95-113.

10. R. J. Gardner and W. F. Pfeffer, Borel measures, Handbook of Set Theoretic Topology, (K. Kunen and J. E. Vaughan, eds.), North-Holland, Amsterdam, 1984, pp. 961-1043.

11. L. Gillman and M. Jerison, Rings of continuous functions, Van Nostrand, Princeton, N.J., 1960.

12. S. P. Gul' ko, On properties of subsets of $\Sigma$-products, Soviet Math. Dokl. 18 (1977), 14381442.

13. E. Hewitt and K. Stromberg, Real and abstract analysis, Springer-Verlag, Berlin and Heidelberg, 1965.

14. A. Kato, Unions of realcompact spaces and Lindelöf spaces, Canad. J. Math. 31 (1979), 12471268.

15. J. D. Knowles, Measures on topological spaces, Proc. London Math. Soc. 17 (1967), 139-156.

16. K. Kuratowski, Topology, vol. I, Academic Press, New York and London, 1966.

17. J. Mack, Directed covers and paracompact spaces, Canad. J. Math. 19 (1967), 649-654.

18. J. Mařik, The Baire and Borel measure, Czechoslovak Math. J. 7 (1957), 248-253.

19. S. Mrówka, Some set-theoretic constructions in topology, Fund. Math. 94 (1977), 83-92.

20. W. Moran, Measures and mappings on topological spaces, Proc. London Math. Soc. 19 (1969), 493-508.

21. K. Morita, Paracompactness and product spaces, Fund. Math. 50 (1962), 223-236.

22. S. Okada and Y. Okazaki, On measure-compactness and Borel measure-compactness, Osaka J. Math. 15 (1978), 183-191.

23. K. A. Ross and A. H. Stone, Products of separable spaces, Amer. Math. Monthly 71 (1964), 398-403.

24. K. A. Ross and K. Stromberg, Baire sets and Baire measures, Ark. Mat. 6 (1965), 151-160.

25. M. E. Rudin, Lectures on set theoretic topology, CBMS Regional Conf. Ser. in Math., vol. 23, Amer. Math. Soc., Providence, R.I., 1975.

26. M. Ulmer, C-embedded L-spaces, Pacific J. Math. 46 (1973), 591-602.

27. R. F. Wheeler, Topological measure theory for completely regular spaces and their projective covers, Pacific J. Math. 82 (1979), 565-584.

28. __ Extensions of a $\sigma$-additive measure to the projective cover, Lecture Notes in Math., vol. 794, Springer-Verlag, Berlin, 1980, pp. 81-104.

29. __ A survey of Baire measures and strict topologies, Exposition Math. 2 (1983), 97-190.

30. R. G. Woods, Ideals of pseudocompact regular closed sets and absolutes of Hewitt realcompactifications, General Topology Appl. 2 (1972), 315-331.

31. A A survey of absolutes of topological spaces, Topological Structures II, Math. Centre Tracts, vol. 116, Math. Centre, Amsterdam, 1979, pp. 323-362.

Faculty of Education, Shizuoka University, Ohya, Shizuoka 422, Japan (Current address of Haruto Ohta)

Faculty of Liberal Arts, Shizuoka University, Ohya, Shizuoka 422, Japan

Current address (Ken-ichi Tamano): Department of Mathematics, Faculty of Engineering, Yokohama National University, 156 Tokiwadai, Hodogaya, Yokohama 240, Japan 